\title{
Neutrophil gelatinase-associated lipocalin immunoexpression in colorectal carcinoma: A stage-specific prognostic factor?
}

\author{
VALERIA BARRESI, ROBERTA LUCIANÒ, ENRICA VITARELLI, AGATA LABATE, \\ GIOVANNI TUCCARI and GAETANO BARRESI
}

Department of Human Pathology, University of Messina, 98125 Messina, Italy

Received July 21, 2010; Accepted September 17, 2010

DOI: $10.3892 / 01.2010 .191$

\begin{abstract}
TNM post-surgical staging is considered to be one of the most powerful prognosticators for colorectal carcinoma. Although patient survival mostly decreases concomitantly to stage increase, in a percentage of cases TNM stage appears only to express the anatomic extent of the neoplasia with no correlation with clinical outcome. Thus, the identification of additional prognostic markers for colorectal cancer is required. Neutrophil gelatinase-associated lipocalin (NGAL) is a 25-kDa protein that appears to play an important role in colorectal cancer progression. In order to evaluate whether NGAL expression may be considered as a predictor of colorectal cancer progression, we analyzed its correlation with clinicopathological characteristics, as well as with patient progression-free survival in a series of surgically resected colorectal carcinomas. A variable NGAL immunoexpression was found in 24 out of the 64 analyzed cases. When only the positive cases were considered, a significant association was found between a high NGAL expression and the presence of distant metastases or high tumor stage. In addition, the presence of NGAL was a significant negative prognostic marker correlated with a shorter progression-free survival in stage I colorectal carcinoma, but not in the remaining TNM stages. If our findings are confirmed in more extensive analyses on stage I colorectal carcinoma, NGAL assessment may be used in order to select those patients with a higher progression risk and to submit them to adjuvant therapies useful to prevent adverse outcome.
\end{abstract}

\section{Introduction}

Colorectal carcinoma is one of the leading causes of cancerrelated death in Western Europe and the US (1). Post-surgical

Correspondence to: Dr Valeria Barresi, Dipartimento di Patologia Umana, Policlinico Universitario G. Martino, Pad D, Via Consolare Valeria, 98125 Messina, Italy

E-mail: vbarresi@unime.it

Key words: neutrophil gelatinase-associated lipocalin, colorectal cancer, prognosis, TNM stage, iron
TNM staging is a prognostic factor that is able to predict the clinical course of this neoplasia, with 5-year survival rates decreasing concomitantly to stage $(2,3)$. For this reason, TNM staging is taken into consideration in the therapeutic protocols applied to colorectal cancer patients. Thus, stage I colorectal carcinoma is treated by surgery alone, with adjuvant chemotherapy being the choice of treatment in order to improve the survival of patients affected by higher stage carcinomas. Nevertheless, in certain instances TNM staging appears only to express the anatomic extent of the neoplasia with no evidence of correlation with patient survival (4). At present, histoprognostic factors able to predict progressionfree and overall survival of colorectal cancer in order to select patients for adjuvant therapies are not available. Thus, factors predicting progression risk, in addition to the currently used clinicalpathological staging system, are required.

Neutrophil gelatinase-associated lipocalin (NGAL) is also known as NRL (neu-related lipocalin), oncogene 24p3, uterocalin and lipocalin 2 . It is a $25-\mathrm{kDa}$ protein that is stored in the granules of human neutrophils (5). Additionally, it belongs to the lipocalin family which consists of more than 50 members, all of which are characterized by the ability to bind and transport small lipophilic substances (6). NGAL was shown to be involved in iron trafficking (7) and to increase the cytoplasmic levels of this mineral (8) by capturing and transporting the iron particles to the inner cell following interaction with specific membrane receptors $(24 \mathrm{p} 3$, megalin). Its role in the delivery of iron to the cells underlies the multiple effects attributed to NGAL. Since it is released by activated neutrophils, this protein is involved in the iron depletion strategy exploited in immune defense against bacterial pathogens (9). Furthermore, NGAL appears to be involved in the growth, development and differentiation of a number of human tissues, as early as in the embryo, via its regulation of iron-responsive genes which are crucial in the differentiation of primordial cells $(10,11)$.

Since NGAL synthesis is induced by factors promoting the development of neoplasias $(6,11,12)$, it was suggested that this protein plays a role in the carcinogenesis and progression of human tumors (11). Accordingly, its overexpression has been found in various types of cancer, including colorectal (13-16). The role of NGAL in colorectal cancer progression (18) is evident due to the existence of a significant association between a high NGAL mRNA quantity and nodal metastases or advanced stage of this neoplasia (17). In addition, the 
induction of NGAL overexpression has been shown to decrease cell-cell adhesion, enhance cell-matrix attachment and increase cell motility and in vitro invasion in colorectal cancer cell lines (18). Subsequently, this study aimed to evaluate whether progression-free survival may differ on the basis of NGAL expression in colorectal cancer and whether this expression is a predictor of disease progression in this neoplasia.

\section{Materials and methods}

Materials. A total of 64 consecutive cases of surgically resected colorectal carcinomas, procured from an equal number of patients (29 female and 35 male patients; age range 36-89 years; mean age 68.09 \pm 12.19 ), were obtained from the files of the Department of Human Pathology of the University of Messina, Italy. None of the patients had received pre-operative chemotherapy for their neoplasia or had colonic carcinoma in situ. Complete tumor resection had been achieved in all cases (R0). A total of 41 cases were located at the left colon (including rectum and sigma), while 23 were located at the right colon (including caecum, ascendant and transversus). Histological grade was established according to the World Health Organization classification system criteria; specifically, 24 cases were grade 1, 36 grade 2, while 4 cases were grade 3 . Of all patients at the time of diagnosis, 20 had lymph nodes and 12 had liver metastases, with 5 of the latter being submitted to surgical resection for their metastases. Follow-up data, including information regarding progression-free survival, were available in all 64 cases. None of the patients with stage I cancer had been treated with adjuvant chemotherapy, while 3 out of 11 patients with stage II colorectal cancer had received chemotherapy (5-fluorouracil plus folinic acid) as had all the patients with stage III or IV cancers.

Methods. All 64 cases were formalin-fixed and paraffinembedded. For each case, $4-\mu \mathrm{m}$ sections were cut from the corresponding paraffin block for subsequent immunohistochemical study.

Briefly, the endogenous peroxidase activity was blocked with $0.1 \% \mathrm{H}_{2} \mathrm{O}_{2}$ in methanol for $20 \mathrm{~min}$. Normal sheep serum was then applied for $30 \mathrm{~min}$ to prevent non-specific adherence of serum proteins. Sections were sequentially incubated at $4^{\circ} \mathrm{C}$ overnight with the primary antibody against NGAL (Santa Cruz Biotechnology, Santa Cruz, CA, USA; 1:100). The bound primary antibody was visualized using the Dako EnVision peroxidase detection system (Dako Cytomation, Glostrup, Denmark), according to the manufacturer's instructions. For immunostaining, the sections were incubated in the dark for 10 min with 3,3'-diaminobenzidine tetra hydrochloride (Sigma Chemical Co., St. Louis, MO, USA), with the amount of $100 \mathrm{mg}$ in $200 \mathrm{ml} 0.03 \%$ hydrogen peroxide in phosphate-buffered saline (PBS). Nuclear counterstaining was performed by Mayer's haemalum. The binding specificity was assessed by omitting the primary antiserum or replacing it with normal rabbit serum or PBS ( $\mathrm{pH}$ 7.4). Proximal tubules within samples of normal renal parenchyma were used as positive controls for the immunoreactions $(15,19)$.

Assessment of the immunostained section was performed by two independent pathologists, who were blinded to the clinicopathological data. NGAL expression was based on the presence of cytoplasmic and membranous staining. The intensity of staining (IS) was graded as negative (0), weak (1), moderate (2) and strong (3). The area of staining positivity (ASP), recorded as a percentage of neoplastic positive cells, was assessed using the values: $0(<10 \%), 1(11-25 \%)$, $2(26-50 \%), 3(51-75 \%)$ and $4(>75 \%)$, according to the procedure previously described $(16,21)$. An intensity distribution (ID) score was then generated for each case by multiplying the values of IS and ASP. Cases with an ID score of 0 were considered to be negative for NGAL.

For the statistical analyses, samples were subdivided into negative and positive tumors, according to the median NGAL ID score, which was 0 . Moreover, when only positive cases were considered, low (ID score 1-2) and high (ID score >2) NGAL expression was defined on the basis of the median ID score of this subgroup (median ID score 2).

Statistical analysis. Fisher's exact and Chi-square tests were performed to assess the statistical correlations between NGAL expression and various clinicopathological characteristics, such as age, gender or the site, histological grade, depth of infiltration $(\mathrm{T})$, presence of nodal $(\mathrm{N})$ or distant metastases $(\mathrm{M})$, as well as tumor stage.

Progression-free survival was assessed by the KaplanMeier method, with the date of primary surgery as the entry data. The end point was characterized as the length of survival to recurrent (local or distant) disease. The Mantel-Cox log-rank test was applied to assess the strength of association between survival time and each of the patient characteristics (age, gender, chemotherapeutic treatment, site, histological grade, TNM stage and NGAL immunoexpression of the tumor) as a single variable. Concomitantly, a multivariate analysis (Cox regression model) was utilised to determine the independent effect of each significant variable.

$\mathrm{P}<0.05$ was considered to be statistically significant. Data were analysed using the SPSS package version 6.1.3 (SPSS Inc., Chicago, IL, USA).

\section{Results}

Table I shows the clinicopathological and immunohistochemical data related to the 64 analyzed colorectal carcinomas. NGAL immunoexpression was found in 24 cases with variable ID scores. Immunostaining was present within the cytoplasm of the neoplastic cells (Fig. 1), whereas no immunolabelling was evident in the normal epithelium adjacent to the tumor. In addition, in positive cases immunoreactivity was always evident at the invasive front of the neoplasm.

No significant associations were found between NGAL presence and the various clinicopathological characteristics (Table II), but when only positive cases were examined, a significant association emerged between a high NGAL expression (ID score $>2$ ) and the presence of distant metastases at the initial diagnosis $(\mathrm{P}=0.017)$ or high $\mathrm{TNM}$ stage $(\mathrm{P}=0.039)$ (Table III).

Post-surgical follow-up ranged between 4 and 117 months. During the follow-up, 24 out of 64 patients developed recurrent disease, with distant metastases in 23 cases and local recurrence in 1 case. Patients who died of independent disease and those with no evidence of disease progression (3/64) were 


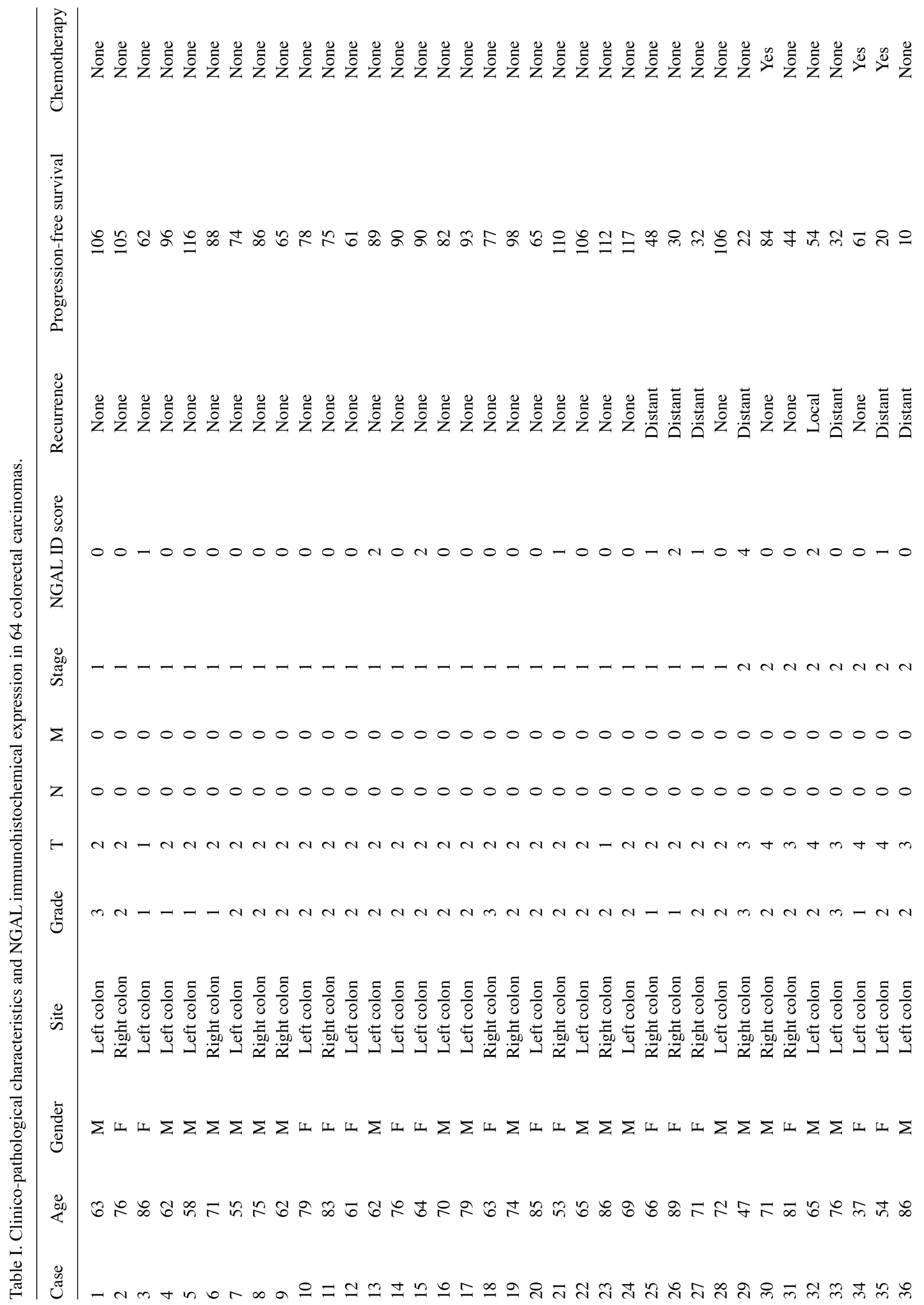




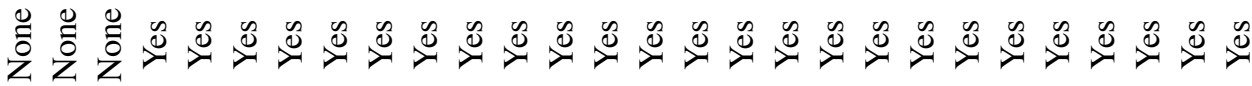

8으윰ำ

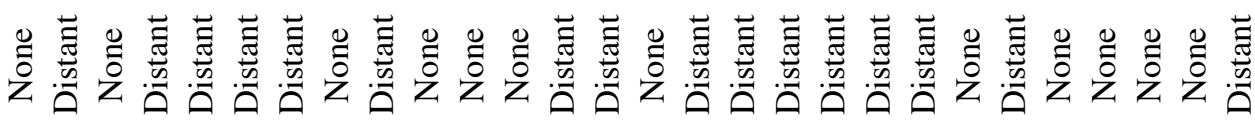

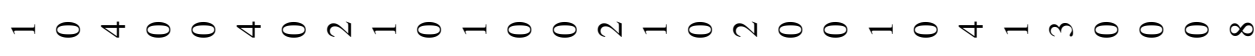

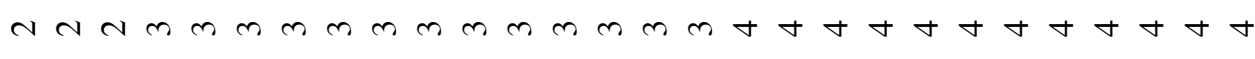

$0000000000000000 \cdots-\cdots \cdots-\cdots \cdots-\cdots-$

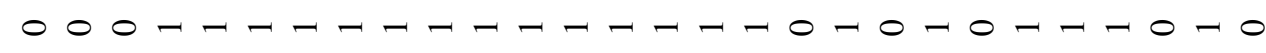

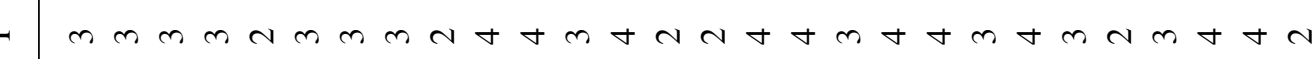

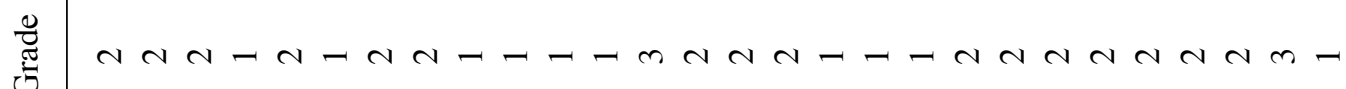

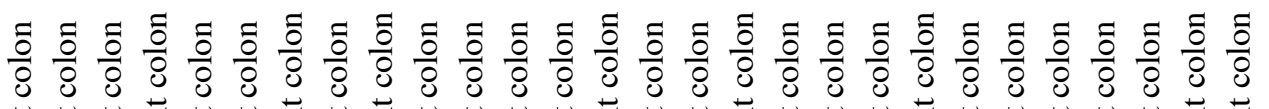

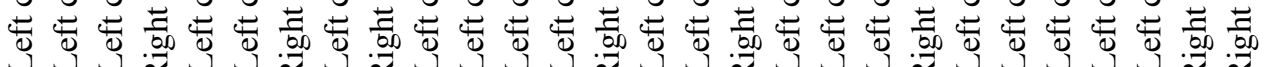

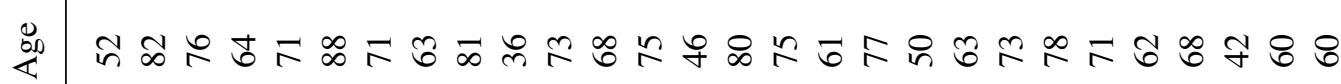

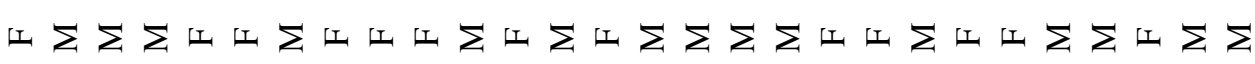



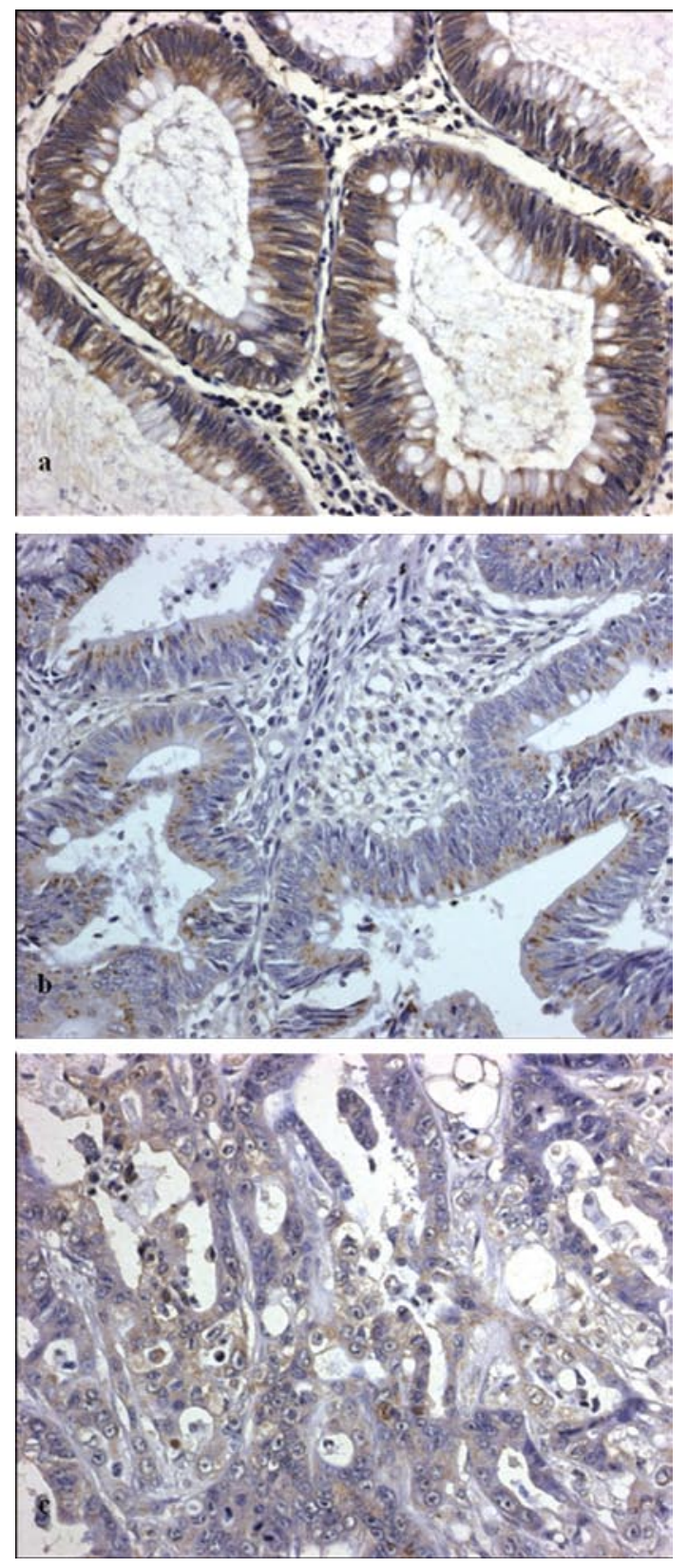

Figure 1. NGAL immunohistochemical expression in the cytoplasm of the neoplastic cells of a (a) well-differentiated (NGAL stain; original magnification, x100), (b) moderately differentiated (NGAL stain; original magnification, x100) and (c) poorly differentiated (NGAL stain; original magnification, $\mathrm{x} 200$ ) colorectal carcinoma.

censored.A total of 23 out of 24 patients with recurrent disease died of disease progression. Univariate survival analyses revealed TNM stage, depth of infiltration, nodal metastases, chemotherapy and NGAL immunoexpression to be significant prognostic factors for progression-free survival (Table IV). In particular, the length of survival to recurrent disease was significantly shorter in those patients with a colorectal carcinoma characterized by a high NGAL expression $(\mathrm{P}=0.0305)$ (Table IV). Nevertheless, the multivariate analysis suggested that NGAL expression was not an independent prognostic factor, whereas the application of chemotherapy was found to be an independent variable as compared to progression-free survival of patients (Table IV). When the prognostic value of
Table II. Statistical correlations between the NGAL immunoexpression and the various clinicopathological characteristics in 24 NGAL-positive colorectal carcinomas.

\begin{tabular}{|c|c|c|c|}
\hline & \multicolumn{2}{|c|}{ NGAL ID score } & \multirow[b]{2}{*}{ P-value } \\
\hline & $1-2$ & $>2$ & \\
\hline Gender & & & 0.192 \\
\hline M & 6 & 4 & \\
\hline $\mathrm{F}$ & 12 & 2 & \\
\hline Age & & & 0.665 \\
\hline$<70$ years & 11 & 3 & \\
\hline$>70$ years & 7 & 3 & \\
\hline Site & & & 1.000 \\
\hline Left colon & 11 & 4 & \\
\hline Right colon & 7 & 2 & \\
\hline Grade & & & 0.201 \\
\hline 1 & 6 & 2 & \\
\hline 2 & 12 & 3 & \\
\hline 3 & 0 & 1 & \\
\hline $\mathrm{T}$ & & & 0.418 \\
\hline 1 & 1 & 0 & \\
\hline 2 & 9 & 2 & \\
\hline 3 & 3 & 3 & \\
\hline 4 & 5 & 1 & \\
\hline $\mathrm{N}$ & & & 1.000 \\
\hline 0 & 11 & 4 & \\
\hline $1-2$ & 7 & 2 & \\
\hline M & & & 0.017 \\
\hline 0 & 16 & 2 & \\
\hline 1 & 2 & 4 & \\
\hline Stage & & & 0.039 \\
\hline 1 & 7 & 0 & \\
\hline 2 & 3 & 1 & \\
\hline 3 & 6 & 1 & \\
\hline 4 & 2 & 4 & \\
\hline
\end{tabular}

ID, intensity distribution; M, male; F, female; TNM, tumor node metastasis.

NGAL expression was specifically investigated according to the TNM stage, the expression of this protein was evidenced as a negative prognostic factor that correlated with a shorter progression-free survival in stage $\mathrm{I}(\mathrm{P}=0.001)$ (Fig. 2), but not in colorectal carcinomas of higher stages (Table $\mathrm{V})$.

\section{Discussion}

NGAL overexpression and correlation with disease progression has been shown in malignancies of the gastro-intestinal tract, including esophageal, gastric and colorectal carcinomas $(17,21,22)$. We investigated for the first time the relationship between NGAL prognostic value and progression-free survival of colorectal carcinoma. In particular, NGAL immunohistochemical expression was evaluated in a series of 64 colorectal carcinomas of different stages and correlated 
Table III. Statistical correlations between NGAL immunoexpression and the various clinicopathological characteristics of the 64 analyzed colorectal carcinomas. ${ }^{a}$

\begin{tabular}{|c|c|c|c|}
\hline & \multicolumn{2}{|c|}{ NGAL ID score } & \multirow[b]{2}{*}{$\mathrm{P}$-value } \\
\hline & 0 & $>0$ & \\
\hline Gender & & & 0.066 \\
\hline $\mathrm{M}$ & 27 & 10 & \\
\hline $\mathrm{F}$ & 13 & 14 & \\
\hline Age & & & 0.438 \\
\hline$<70$ years & 18 & 14 & \\
\hline$>70$ years & 22 & 10 & \\
\hline Site & & & 1.000 \\
\hline Left colon & 26 & 15 & \\
\hline Right colon & 14 & 9 & \\
\hline Grade & & & 0.055 \\
\hline 1 & 9 & 8 & \\
\hline 2 & 26 & 5 & \\
\hline 3 & 5 & 1 & \\
\hline $\mathrm{T}$ & & & 0.932 \\
\hline 1 & 1 & 1 & \\
\hline 2 & 21 & 11 & \\
\hline 3 & 10 & 6 & \\
\hline 4 & 8 & 6 & \\
\hline $\mathrm{N}$ & & & 0.419 \\
\hline 0 & 29 & 15 & \\
\hline $1-2$ & 11 & 9 & \\
\hline M & & & 1.000 \\
\hline 0 & 30 & 18 & \\
\hline 1 & 10 & 6 & \\
\hline Stage & & & 0.201 \\
\hline 1 & 21 & 7 & \\
\hline 2 & 4 & 4 & \\
\hline 3 & 5 & 7 & \\
\hline 4 & 10 & 6 & \\
\hline
\end{tabular}

ID, intensity distribution; M, male; F, female; TNM, tumor node

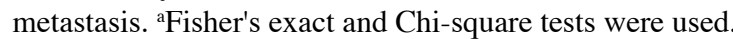

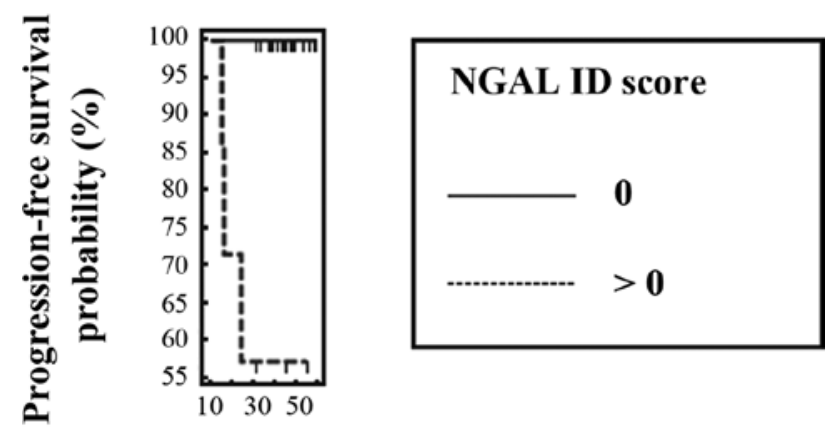

DFS(months)

Figure 2. Kaplan-Meier-specific progression-free survival curve in relation to NGAL expression in stage I colorectal carcinomas. A significantly shorter progression-free survival was noted in cases with NGAL expression comparison to cases with no NGAL immunoexpression. DFS, disease-free survival.
Table IV. Univariate and multivariate analyses for progressionfree survival in 64 patients affected by colorectal carcinoma.

A, Univariate analysis.

\begin{tabular}{lccc}
\hline Charateristics & $\chi^{2}$ & Df & P-value \\
\hline Age & 0.9862 & 1 & 0.3207 \\
Gender & 0 & 1 & 0.9935 \\
Site & 1.1595 & 1 & 0.2816 \\
Grade & 2.2580 & 2 & 0.3234 \\
T & 9.1260 & 3 & 0.0088 \\
N & 7.3205 & 1 & 0.0068 \\
M & 3.3093 & 1 & 0.0689 \\
Stage & 16.2523 & 3 & 0.0010 \\
Chemotherapy & 8.8210 & 1 & 0.0030 \\
NGAL immunoexpression & 4.6795 & 1 & 0.0305 \\
\hline
\end{tabular}

B, Multivariate analysis.

\begin{tabular}{lcccc}
\hline Variable & $\beta$ & SE & $\operatorname{Exp}(\beta)$ & P-value \\
\hline Chemotherapy & 1.2116 & 0.4348 & 3 & 0.005 \\
\hline
\end{tabular}

df, degrees of freedom; SE, standard error.

Table V. NGAL univariate analysis for progression-free survival according to TNM staging of 64 colorectal carcinomas.

\begin{tabular}{llll}
\hline $\begin{array}{l}\text { Univariate analysis } \\
\text { (Characteristic: NGAL expression) }\end{array}$ & $\chi^{2}$ & Df & P-value
\end{tabular}

\begin{tabular}{lrrr}
\hline Stage & & & \\
I & 10.5775 & 1 & 0.0011 \\
II & 0.0079 & 1 & 0.9290 \\
III & 0.2400 & 1 & 0.6242 \\
IV & 0.1811 & 1 & 0.6704
\end{tabular}

Df, degrees of freedom; TNM, tumor node metastasis.

with the various clinicopathological characteristics, as well as with progression-free survival of the patients.

A variable NGAL expression was found in 24 out of 64 cases. In accordance with previous reports (16-18), NGAL immunoexpression was found in the neoplastic cells of positive cases, but not in the adjacent normal epithelium, in line with its suggestive role in colorectal carcinogenesis. Immunostaining was localized at the cytoplasm of the neoplastic cells, as in the case of breast cancer (13). This localization suggests NGAL internalization to the inner cell following its binding to membrane receptors. Since NGAL is involved in iron uptake, we hypothesize that NGAL expression and cytoplasmic localization reflect augmented iron requirement by the neoplastic elements of colorectal carcinoma, as previously described (18). Notably, NGAL appears to play a role in carcinogenesis by 
promoting iron uptake from the extracellular space within the malignant cells, a fundamental process for the maintenance of neoplastic cell multiplication (14,23). Besides, it is well-known that iron overload promotes carcinogenesis through the induction of oxidative DNA damage (24), whereas iron depletion leads to cell cycle arrest and apoptosis (25).

Notably, NGAL staining was mainly observed in the invasive front of the tumor in positive cases. This may be related to its ability to increase the invasive potential of tumor cells. Studies have shown that NGAL forms a complex with matrix metalloproteinase-9 (MMP-9), preventing MMP-9 degradation and resulting in increased MMP-9 enzyme activity in vitro (26). MMP-9 plays a role in cancer progression by degrading the molecular components of the basement membrane and extracellular matrix, releasing vascular endothelial cell growth factor (VEGF) from the extracellular matrix, and enabling angiogenesis, invasion and distant metastasis (26-28). Thus, NGAL may play a role in cancer progression by causing increased MMP-9 enzymatic activity, thereby promoting the invasive and metastatic potential of cancer cells in vivo and in vitro (28). However, NGAL was recently shown to increase invasiveness of colorectal cancer cells through an MMP-9-independent process (18). It has been shown that NGAL overexpression enhances the invasive and metastatic potential of colon cancer cells by decreasing e-cadherin-mediated cell-cell adhesion through an iron-dependent mechanism (18). In contrast to other authors (17), we failed to demonstrate any significant correlation between NGAL and the depth of infiltration of tumors. Nevertheless, the role of NGAL in the invasiveness of colorectal carcinoma was confirmed by a significant association between a high NGAL expression and the presence of distant metastases or a high TNM stage at the time of initial diagnosis of the analyzed colorectal carcinomas. Notably, NGAL involvement in the development of metastases had previously been hypothesized, since inhibition of this protein results in the blockage of lung metastases from breast carcinoma (29).

NGAL overexpression was previously considered to be a negative prognostic factor in colorectal cancer due to its significant association with the presence of adverse factors, such as lymph node metastases or venous invasion (17). However, its impact on specific survival in relation to neoplasia has yet to be investigated. Our results showed that patients with tumors characterized by NGAL immunohistochemical expression exhibited a significantly shorter progression-free survival in comparison to patients with absent NGAL staining. Thus, NGAL appears to act as a negative prognostic factor for colorectal cancer, as in the case of breast cancer (13). Notably, when the relationship between NGAL predictive value and disease-progression risk was evaluated according to the TNM stage of neoplasias, the presence of NGAL appeared to be a significant predictor of progression risk in stage I colorectal carcinoma, but not in the remaining TNM stages. Only the former group of patients had not been submitted to chemotherapy, which emerged as an independent prognostic factor for progression-free survival in our cohort.

In conclusion, the present study showed that NGAL immunoexpression is a negative prognostic marker associated with shorter disease-specific survival in stage I colorectal carcinomas. If our findings are confirmed on larger cohorts of stage I colorectal carcinomas, NGAL assessment in colon cancer tissue may be used as a prognostic tool to determine tumors with a higher progression risk, so that selected patients may undergo adjuvant treatments in order to prevent adverse outcome. Moreover, the inhibition of NGAL expression or function may represent the target for novel therapeutic strategies in order to prevent disease progression in NGAL-positive colorectal carcinomas. Colorectal carcinomas showing NGAL expression may benefit from therapies with iron chelators as anti-cancer agents. Notably, since iron deprivation caused by iron chelators inhibits cell proliferation, iron and its corresponding transport proteins have become new targets in cancer therapy (30,31). Finally, since the efficacy of anti-NGAL antibodies has been shown by blocking cancer metastasis in mice (29), NGAL expression and involvement in colorectal cancer progression and metastatic disease may be exploited for such novel anti-cancer therapies.

\section{References}

1. Landis H, Murray T, Bolden S and Wingo PA: Cancer statistics. Cancer J Clin 49: 8-31, 1999.

2. Wiggers T, Arends JW, SchutterB, Volovics L and Bosman FT: A multivariate analysis of pathologic prognostic indicators in large bowel cancer. Cancer 61: 386-389, 1988.

3. Wu XC, Chen VW, Steele B, Ruiz B, Fulton J, Liu L, Carozza SE and Greenlee R: Subsite-specific incidence rate and stage of disease in colorectal cancer by race, gender, and age group. Cancer 92: 2547-2554, 2001.

4. Di Gregorio C, Benfatti P, Losi L, Roncucci L, Rossi G, Ponti G, Marino M, Pedroni M, Scarselli A, Roncari B and Ponz de Leon M: Incidence and survival of patients with Dukes' A (stages T1 and T2) colorectal carcinoma: a 15-year population-based study. Int J Colorectal Dis 20: 147-154, 2005.

5. Cowland JB and Borregaard N: Molecular characterization and pattern of tissue expression of the gene for neutrophil gelatinaseassociated lipocalin from humans. Genomics 45: 17-23, 1997.

6. Bratt T: Lipocalins and cancer. Biochim Biophys Acta 1482: 318-326, 2000.

7. Yang J, Goetz D, Li JY, Wang W, Mori K and Setlik D: An iron delivery pathway mediated by a lipocalin. Mol Cell 10: 1045-1056, 2002.

8. Goetz DH, Willie ST, Arme RS, Bratt T, Borregaard N and Strong RK: Ligand preference inferred from the structure of neutrophil gelatinase associated lipocalin. Biochemistry 39: 1935-1941, 2000.

9. Goetz DH, Holmes MA, Borregaard N, Bluhm ME, Raymond KN and Strong RK: The neutrophil lipocalin NGAL is a bacteriostatic agent that interferes with siderophore-mediated iron acquisition. Mol Cell 10: 1033-1043, 2002.

10. Gwira JA, Wei F, Ishibe S, Ueland JM, Barasch J and Cantley LG: Expression of neutrophil associated-gelatinase lipocalin regulates epithelial morphogenesis in vitro. J Biol Chem 280: 7875-7882, 2005

11. Bolignano D, Donato V, Lacquaniti A, et al: Neutrophil gelatinase-associated lipocalin (NGAL) in human neoplasias: a new protein enters the scene. Cancer Lett 288: 10-16, 2010.

12. Hannai J, Mammoto T, Seth P, Mori K, Karumanchi SA, Barasch J and Sukhatme VP: Lipocalin 2 diminishes invasiveness and metastasis of Ras-transformed cells. J Biol Chem 280: 3641-3647, 2005.

13. Bauer M, Eickhoff JC, Gould MN, Mundhenke C, Maas N and Friedl A: Neutrophil gelatinase-associated lipocalin (NGAL) is a predictor of poor prognosis in human primary breast cancer. Breast Cancer Res Treat 108: 389-397, 2008.

14. Iannetti A, Pacifico F, Acquaviva R, et al: The neutrophil gelatinase-associated lipocalin (NGAL), a NF-kappaB-regulated gene, is a survival factor for thyroid neoplastic cells. Proc Natl Acad Sci USA 105: 14058-14063, 2008.

15. Barresi V, Ieni A, Bolignano D, Magno C, Buemi $M$ and Barresi G: Neutrophil gelatinase-associated lipocalin immunoexpression in renal tumors: correlation with histotype and histological grade. Oncol Rep 24: 305-310, 2010. 
16. Nielsen BS, Borregaard N, Bundgaard JR, Timshel S, Sehested M and Kjeldsen L: Induction of NGAL synthesis in epithelial cells of human colorectal neoplasia and inflammatory bowel diseases. Gut 38: 414-420, 1996.

17. Zhang XF, Zhang Y, Zhang XH, Zhou SM, Yang GG, Wang OC, Guo GL, Yang GY and $\mathrm{Hu}$ XQ: Clinical significance of Neutrophil gelatinase-associated lipocalin (NGAL) expression in primary rectal cancer. BMC Cancer 91: 34, 2009.

18. Hu L, Hittelman W, Lu T, Ji P, Arlinghaus R, Shmulevich I, Hamilton SR and Zhang W: NGAL decreases E-cadherinmediated cell-cell adhesion and increases cell motility and invasion through Rac1 in colon carcinoma cells. Lab Invest 89: 531-548, 2009.

19. Friedl A, Stoesz SP, Buckley P and Gould MN: Neutrophil gelatinase-associated lipocalin in normal and neoplastic human tissues. Cell type-specific pattern of expression. Histochem J 31: 433-441, 1999.

20. Moniaux N, Chakraborty S, Yalniz M, et al: Early diagnosis of pancreatic cancer: neutrophil gelatinase-associated lipocalin as a marker of pancreatic intraepithelial neoplasia. Br J Cancer 98: 1540-1547, 2008.

21. Kubben FJ, Sier CF, Hawinkels LJ, et al: Clinical evidence for a protective role of lipocalin-2 against MMP-9 autodegradation and the impact for gastric cancer. Eur J Cancer 43: 1869-1876, 2007.

22. Zhang H, Xu L, Xiao D, et al: Upregulation of neutrophil gelatinase-associated lipocalin in oesophageal squamous cell carcinoma: significant correlation with cell differentiation and tumour invasion. J Clin Pathol 60: 555-561, 2007.

23. Devireddy LR, Gazin C, Zhu X and Green MR: A cell-surface receptor for lipocalin $24 \mathrm{p} 3$ selectively mediates apoptosis and iron uptake. Cell 23: 1293-1305, 2005.
24. Toyokuni S: Role of iron in carcinogenesis: cancer as a ferrotoxic disease. Cancer Sci 100: 9-16, 2009.

25. Le NTV and Richardson DN: The role of iron in cell cycle progression and the proliferation of neoplastic cells. Biochim Biophys Acta 1603: 3146, 2002.

26. Fernandez CA, Yan L, Louis G, Yang J, Kutok JL and Moses MA: The matrix metallo-proteinase-9/neutrophil gelatinase associated lipocalin complex plays a role in breast tumor growth and is present in the urine of breast cancer patients. Clin Cancer Res 11: $5390-5395,2005$.

27. Lee S, Jilani SM, Nikolova GV, Carpizo D and Iruela-Arispe M: Processing of VEGF-A by matrix metalloproteinases regulates bioavailability and vascular patterning in tumors. J Cell Biol 169: 681-691, 2005.

28. Yan L, Borregaard N, Kjeldsen L and Moses MA: The high molecular weight urinary matrix metalloproteinase (MMP) activity is a complex of gelatinase B/MMP-9 and neutrophil gelatinase-associated lipocalin (NGAL). Modulation of MMP-9 activity by NGAL. J Biol Chem 276: 37258-37265, 2001.

29. Leng X, Ding T, Lin H, et al: Inhibition of lipocalin 2 impairs breast tumorigenesis and metastasis. Cancer Res 69: 8579-8784, 2009.

30. Buss JL, Greene BT, Turner J, Torti FM and Torti SV: Iron chelators in cancer chemotherapy. Curr Top Med Chem 4: 1623-1635, 2004.

31. Jones DT, Trowbridge IS and Harris AL: Effects of transferrin receptor blockade on cancer cell proliferation and hypoxiainducible factor function and their differential regulation by ascorbate. Cancer Res 66: 2749-2756, 2006. 